\title{
Experimental and model-based study of the robustness of line-edge roughness metric extraction in the presence of noise
}

\author{
Patrick P. Naulleau, ${ }^{1}$ Jason P. Cain ${ }^{2}$ \\ ${ }^{1}$ Center for X-Ray Optics, Lawrence Berkeley National Laboratory, Berkeley, CA 94720 \\ ${ }^{2}$ EECS Department, University of California, Berkeley, CA 94720
}

\begin{abstract}
As critical dimensions shrink, line edge and width roughness (LER and LWR) become of increasing concern. Crucial to the goal of reducing LER is its accurate characterization. LER has traditionally been represented as a single rms value. More recently the use of power spectral density (PSD), height-height correlation (HHCF), and $\sigma$ versus length plots has been proposed in order to extract the additional spatial descriptors of correlation length and roughness exponent. Here we perform a modeling-based noise-sensitivity study on the extraction of spatial descriptors from line-edge data as well as an experimental study of the robustness of these various descriptors using a large dataset of recent extreme-ultraviolet exposure data. The results show that in the presence of noise and in the large dataset limit, the PSD method provides higher accuracy in the extraction of the roughness exponent, whereas the HHCF method provides higher accuracy for the correlation length. On the other hand, when considering precision, the HHCF method is superior for both metrics.
\end{abstract}

Keyword: extreme ultraviolet, lithography, multilayer, mask, defects 


\section{Introduction}

Line edge and width roughness (LER and LWR), has become an issue of increasing concern as projection lithography techniques push to smaller and smaller feature sizes. This poses significant challenges to the development of photo-resist for next-generation lithography techniques such as 193-nm immersion and extreme-ultraviolet (EUV) lithography. For example at the 32-nm DRAM half-pitch fabrication node, the International Technology Roadmap for Semiconductors [1] calls for a resist contribution to LWR of less than $1.7 \mathrm{~nm}$, which in turn requires even finer characterization capabilities to develop such resists. Representation of the LER, however, as a single $3 \sigma$ rms number may not be adequate to address the needs of the nano-electronics industry. To some extent this has been addressed by the ITRS by specifying the frequency range for the roughness, however, still more descriptive metrics may be required. Additional metrics could also prove beneficial for fundamental learning about resists.

To address the potential problem of inadequate LER characterization, several additional metrics have been proposed [2-4]. Of particular interest are metrics that provide insight into the spatial frequency content of the roughness such as correlation length and roughness exponent. Various methods may be used to estimate these metrics including the characterization of the power spectral density (PSD) [5], the height-height correlation function (HHCF) [3], and the roughness versus length (SVL) plot [2]. It has been shown in the literature [6] that the height-height correlation function is the preferable method for estimating these parameters. This choice is due primarily to the high noise sensitivity of the PSD method. The HHCF method has also been analyzed from the perspective of sensitivity to image filtering parameters, with optimal settings based on selecting a filter size that renders metric extraction relatively insensitive to incremental changes in filter parameters [3]. The question of accuracy of spatial metric extraction in the presence of noise and noise-reduction 
techniques has not been addressed. Here we present the results from a modeling based study of these questions. Moreover, a large set of experimental data is analyzed quantifying the precision of the various methods in a real world situation. Knowledge of this precision is crucial when trying to use a particular metric to compare different resists or process conditions.

\section{Metric extraction accuracy}

Before proceeding to the characterization of reproducibility on experimental data, we verify the accuracy of the extraction algorithms on ideal model data. Metric extraction is performed using fully automated algorithms using offline image analysis software. We note that detailed mathematical descriptions of the PSD, HHCF, and SVL functions used here and the extraction of spatial parameters such as correlation length and roughness exponents from these functions can readily be found in the literature. References in this area specifically dealing with resist LER include $[2-4,6]$. As is typically done in the literature, we assume the line-edge residuals to be well described in terms of self-affine fractal scaling. Following Refs. [3,7], we choose the fractal process to have an autocorrelation function $\Gamma(x)$ defined as

$$
\Gamma(x)=\sigma^{2} \exp \left[-\left(x / L_{c}\right)^{2 \alpha}\right]
$$

where $\sigma^{2}$ is the variance, $L_{c}$ is the correlation length, and $\alpha$ is the roughness exponent. The selfaffine sequence is generated through spectral shaping methods [8] where the target autocorrelation function [Eq. (1)] is Fourier transformed to generate the target power spectrum, which in turn is used to shape the spectrum of a random number series.

Using this technique we generate line edges, each comprised of 1024 points with 2-nm sample spacing, and extract roughness exponent and correlation length metrics using the PSD, HHCF, and SVL methods, respectively. We note that the correlation length reported for the PSD 
method is actually computed from the autocorrelation function (the Fourier transform of the PSD) using the equivalent top-hat metric [9]. Using this method allows the correlation length information to be used as a priori information in the extraction of roughness exponent from the PSD. We note that we have not attempted to use the SVL plot to extract correlation length data because long line lengths are required and the method provides limited accuracy. For improved statistics, a total of 216 line edges are generated and the relevant computed functions averaged over all line edges. The metrics are then extracted from the averaged PSD, HHCF, and SVL functions, respectively. We consider roughness exponents ranging from 0.1 to 0.9 at a correlation length of $25 \mathrm{~nm}$ [Figs. 1(a) and (b)], and a range of correlation lengths (15 to $50 \mathrm{~nm}$ ) at a fixed roughness exponent of 0.5 [Figs. 1(c) and (d)].

The results show that in terms of the performance being independent of roughness exponent, on average the HHCF method performs best, although all three method perform fairly well for roughness exponent values greater than 0.2. Considering instead the dependence on correlation length, we see that the PSD method slightly outperforms the HHCF method, especially in terms of the extraction of the correlation length. We note that the HHCF and SVL results in Fig. 1 differ slightly in the details from Ref. [6], however, the general conclusions regarding metric extraction accuracy are basically the same. These differences are presumably due to variations in the implementations of the metric extraction computations.

\section{Noise sensitivity}

Having verified the accuracy of the extraction algorithms on ideal model data, we now turn to characterizing the precision and accuracy of these various algorithms on in the presence of noise. Following Ref. [4], we assume noise in the measurement process to be represented as additive white Gaussian noise on the true edge position data. We can thus simply add Gaussian noise to 
the ideal model edge data from above and observe its effect on metric extraction characteristics.

In all the noise studies below we choose to work with a rms roughness of $1 \mathrm{~nm}$, a roughness exponent of 0.75 , and a correlation length of $25 \mathrm{~nm}$. We consider noise levels ranging from 0.1 to $0.9 \mathrm{~nm}$ rms. Figure 2 shows PSDs for the two extremes of the added Gaussian noise as well as the noise-free case. As is typically seen in experimental data, the plots with noise in Fig. 2 display an evident noise floor caused by the additive white Gaussian noise overriding the $1 / \mathrm{f}$ behavior of the self-affine edge data at high frequencies.

Figure 3 shows the fractional error in the extracted roughness exponent (a) and the correlation length (b) as a function of noise magnitude. As above, the results are based on averaging over 216 line edges. In terms of accuracy in the presence of noise, we find the PSD method to be the best choice for the roughness exponent and the HHCF to be the best choice for the correlation length. The superior performance of the PSD method in the extraction of the roughness exponent is likely due to the natural noise reduction provided by PSD method, which relies on only a small segment of the frequency domain to determine the roughness exponent whereas the additive noise is white. Given that the power spectrum of the true edge is highly concentrated, such a filtering gain should also be applicable to the HHCF and SVL methods, as well as the extraction of the correlation length from the PSD (or more precisely, its Fourier transform). This can be done by lowpass filtering the line edge data keeping only those terms above the noise floor. This filtered line edge data can then be used to compute the HHCF, SVL, and autocorrelation functions. Doing so yields the results in Fig. 4 where we see much improved performance in roughness exponent extraction (a) for both the HHCF and SVL methods making them quite comparable to the PSD method in terms of total variation as a function of noise. This filtering technique is also seen to provide dramatic improvement in the correlation length [Fig. 
4(b)] extraction using both the HHCF and PSD methods. The HHCF method still slightly outperforms the PSD method in terms of correlation length accuracy.

It is also important to consider precision as a function of noise. The results above are based on averaging over a total of 216 line edges; however in practice, this may not be readily feasible. To gauge the precision, we take the same 216 realization data sets described above and compute the roughness metrics for each edge individually. We then take the standard deviation of the computed metrics over the 216 samples (Fig. 5). The standard deviation for the roughness exponent [Fig. 5(a)] is measured relative to the averaged value over the 216 line edges. We see that both the HHCF and SVL methods outperform the PSD method in terms of precision for noise levels of $0.7 \mathrm{~nm}$ and below. The HHCF continues to perform well at even higher noise levels, whereas the SVL method degrades: on average the uncertainty on the roughness exponent computed from the PSD is $41 \%$, whereas the uncertainty from the HHCF and SVL methods are only approximately $16 \%$ and $27 \%$, respectively. For the correlation length, the standard deviation is reported as an absolute value in $\mathrm{nm}$, where the nominal value for the correlation length is $25 \mathrm{~nm}$. Again the HHCF, with an average standard deviation of $4.5 \mathrm{~nm}$, performs much better than the PSD method having an average standard deviation of $11.9 \mathrm{~nm}$. We also see the surprising result that the correlation length precision is nominally independent of noise level. We note that the precision data presented above was based on filtered line-edge data which was shown in Fig. 4 to improve the accuracy. It is also important to realize that the precision numbers presented here are based on the measurement of a single edge. If we assume the roughness on all edges to be uncorrelated, it is evident that averaging over multiple edges will improve the precision as $\operatorname{sqrt}(\mathrm{N})$ where $\mathrm{N}$ is the total number of edges averaged.

\section{Image noise modeling}


In the noise modeling above, noise is only treated at the extracted line-edge level making it impossible to consider the effect of image-based filtering on accuracy and precision. To address this, we take model edge data and synthesize images to which we can add noise. The images are synthesized with subpixel edge position resolution by implementing smooth sigmoidal transitions from bright to dark at each edge. Figure 6 shows an example synthesized ideal image comprised of a series of lines with self-affine edges. In this case the line pitch is $270 \mathrm{~nm}$, the LER is $10 \mathrm{~nm}$, the roughness exponent is 0.75 , and the correlation length is $25 \mathrm{~nm}$. The large LER value is used for illustrative purposes.

To verify the accuracy of both the synthesis of line images and edge extraction algorithms used to analyze the image data, we generate 8 independent images each comprised of 6 independent lines, yielding a total of 96 edges. All the edges are generated with a roughness exponent of 0.75 , a correlation length of $25 \mathrm{~nm}$, and an LER of $3 \mathrm{~nm}$. The pixel size in the generated image is $2 \mathrm{~nm}$ square and the image size $1024 \times 1024$. The image analysis is again performed using offline image analysis software. The line-edge PSD recovered from the synthesized image data is shown in Fig. 7 displaying good behavior throughout the entire frequency range. Considering the spatial metrics: the PSD method recovers a roughness exponent of 0.72 and a correlation length of the $29 \mathrm{~nm}$ and the HHCF method recovers a roughness exponent of 0.75 and a correlation length of $24 \mathrm{~nm}$. The SVL method does not perform quite as well, yielding a roughness exponent of 0.62 .

Having verified the accuracy of the image synthesis and edge extraction algorithms, we next turn to studying the impact of image-based noise. Noise is now treated as additive Gaussian white noise on the image instead of on the edge data as in the previous section. Figure 8 shows a representative synthesized image with LER and additive Gaussian noise where the standard 
deviation of the noise is $10 \%$ the full magnitude of the underlying synthesized image. Figure 9 shows the corresponding PSD displaying noise floor behavior similar to that observed in the addition of edge noise (Fig. 2). Figure 10 shows the metrics recovered from the synthesized images as a function of added noise. The noise magnitude is defined as follows: a noise magnitude of 0.1 corresponds to the addition of Gaussian white noise with a standard deviation equal to $10 \%$ of the full magnitude of the noise-free lines. The off-line software finds the edge locations to sub-pixel accuracy by linear interpolation and determining where the interpolated edge crosses the threshold which is set to $50 \%$.

Figures 10(a) and 10(b) show the measurement results obtained without filtering the extracted line-edge data to depend strongly on the noise level. Alternatively filtering the lineedge data [Figs. 10(c) and 10(d)] improves the situation and we see that for the case of correlation length, virtually no dependence on noise remains. As we saw in the case of the noise added directly to the line-edge data, the HHCF method performs better in terms of accuracy in the presence of noise for the extraction of the correlation length and the PSD performs better for the extraction of the roughness exponent in the presence of noise.

Figure 11 shows precision values as a function of noise for the image-noise case. As seen for noise added directly to the line-edge data, the precision is superior for the HHCF and SVL methods compared to the PSD method. We also again see the correlation length precision results to be relatively independent of noise magnitude.

Now defining the noise directly in image space it is also possible to consider the effect of image-space filtering on accuracy and precision. We begin by studying the effect of the filter alone (no image noise). As above, the roughness exponent of the model line edge is 0.75 and the correlation length $25 \mathrm{~nm}$. Figures 12 and 13 show the results from a two-dimensional parametric 
study considering the $x$ and $y$ filter sizes, where the lines are defined to run parallel to the $y$ axis. For each filter setting a total of 96 line edges are analyzed as described above. The filter is Gaussian with a sigma equal to one third the total filter size in pixels for each axis independently. The Fig.12 results showing the error in the roughness exponent extraction demonstrate that filtering in the $x$ direction has virtually no effect on the quality of the metric extraction, whereas filtering in the $y$ direction considerably impacts the measurement accuracy. This trend is independent of the extraction method used, however, the PSD method is slightly more tolerant to the $y$ filter size. Similar results are seen in Fig. 13 for the correlation length, however, in this case the HHCF method demonstrates less sensitivity to the $y$ filter size.

Having studied the impact of image-space filtering on metric extraction accuracy in the absence of noise, we next turn to the case with image noise. For the examples below we assume an image noise level of 0.05 , (see definition above) corresponding to a noise floor approximately two orders of magnitude down from the PSD peak. This level is chosen because it corresponds well to the noise level observed in the experimental data discussed below. Figure 14 shows the extracted roughness exponent error. In all cases, image-space filtering shows beneficial effects in dealing with image noise. This benefit can be obtained with $\mathrm{x}$ filtering and thus is not simply a balancing of the noise-induced error with filter induced error as is the case for $y$ filtering. Figure 15 shows accuracy results for the extracted correlation length. Filtering in the $x$ direction provides virtually no benefit for this term and filtering in the $y$ direction is strongly detrimental.

In Figs. 16 and 17 we show the precision results. For precision on the extracted roughness exponent (Fig. 16), all three methods benefit slightly from $x$ filtering whereas $y$ filtering significantly degrades the precision. The results are very similar for the extracted correlation length, however, in this case $x$ filtering has virtually no effect. In summary, image- 
space filtering in the direction orthogonal to the lines is beneficial both in terms of accuracy and precision, whereas filtering in the direction of the lines has a negative impact.

Finally we consider which of the two filtering techniques used above (line-edge data filtering and image-space filtering) is more effective. Figure 18 shows the metric extraction accuracy as a function of noise with image-space filtering alone [Figs. 18(a) and (b)] and combined with line-edge data filtering [Figs. $18(\mathrm{c})$ and (d)]. The image-space filter is $12 \times 1$ pixels (orthogonal to lines) with a $\sigma$ of 4 . Comparing to the results in Fig. 10, we see that imagespace filtering alone is minimally effective compared to line-edge data filtering. The one exception is for the extraction of the roughness exponent using the PSD method where imagespace filtering seems to outperform line-edge data filtering. In general, the best results are achieved by combining the two methods. Combining the two methods is also beneficial for the precision as seen in Fig. 19.

\section{Experimental description}

Having verified the accuracy of the extraction algorithms on model data, we now turn to characterizing the precision of these various metric extraction algorithms on experimental EUV data. The exposures used were obtained on the EUV micro-exposure tool installed at Lawrence Berkeley National Laboratory. This synchrotron-based exposure tool [10] operates at a wavelength of $13.5 \mathrm{~nm}$ and has a numerical aperture of 0.3 , providing for sub-30-nm resolution. The wafer was exposed with 100 identical die to provide the requisite data set. With a field size of only $200 \times 600 \mu \mathrm{m}$ at the wafer, these 100 die fit within an area smaller than $25 \mathrm{~mm}^{2}$. The benefit of using such a small area is that the process parameters (resist thickness, post application and exposure bakes, development, ...) can be assumed to be stable over the complete data set. The features used in this study were all of $60-\mathrm{nm}$ lines and spaces printed in a $125-\mathrm{nm}$ thick layer 
of Rohm and Haas $M E T-1 K$ resist (XP 3454C). This feature size falls well within the resolution capabilities of $M E T-1 K$ resist [10], further reinforcing the stability of the experimental data. Resist line images were collected using a Hitachi 4800 SEM and analyzed off line.

For the purpose of this analysis, it is assumed that the true LER metrics of interest are constant over the entire dataset and all that is varying is the noise. Under this assumption, the metric extraction method providing the least variation over the dataset is assumed to be optimal in terms of precision and thus practical utility. We choose the analysis conditions to match those yielding the highest accuracy based on model data: image-space Gaussian filtering with a $12 \times 1$ pixel size (orthogonal to lines) and a $\sigma$ of 4 in addition to line-edge data filtering.

Figure 20 shows a representative SEM image from the dataset. The images are taken at a magnification of 150,000 capturing approximately 5 lines. Figure 21 shows the LER PSD averaged over the entire dataset (1030 edges). A significant noise floor is evident arising from metrology noise, which can be observed as typical SEM "snow" in Fig. 20. The metrics extracted from the 1030 edge dataset is shown in Table 1. Based on the findings above, we assume the filtered values (both image-space and edge data combined) to be the most accurate. Based on sensitivity to filtering, the PSD method appears to be superior for the extraction of the roughness exponent, whereas the HHCF is superior for correlation length. In practice this could be important since it indicates more latitude in the specifics of the filter settings and potentially more robustness in the presence of noise.

Next we consider precision for the experimental data. The precision is determined based on the variation of the extracted metrics from image to image. For each analyzed image all the line edges present in the image are averaged. The typical number of analyzed lines in each image is 5 . Table 2 shows the results. The roughness exponent $(\alpha)$ precision is fractional relative to the 
corresponding average extracted value from Table 1 and the correlation length $\left(L_{c}\right)$ is absolute in nm. As seen with the model data, the HHCF and SVL methods outperform the PSD method in precision, especially for the roughness exponent. With filtering, the PSD method shows a precision of only $28 \%$ when analyzing a single image for roughness exponent and $4.6 \mathrm{~nm}$ for the correlation length. The HHCF method on the other hand has $9 \%$ precision for the roughness exponent and $2.5 \mathrm{~nm}$ for the correlation length.

\section{Discussion}

A detailed study of the noise sensitivity and filtering effectiveness for various LER metrics has been performed. The results show that in the presence of noise and large dataset limit, the PSD method provides higher accuracy in the extraction of the roughness exponent, whereas the HHCF method provides higher accuracy for the correlation length. On the other hand, when considering precision, the HHCF method is superior for both metrics.

Both extracted line edge and image-based filtering was found to be beneficial for both accuracy and precision. The filtering also was found not to change the relative performance of the methods. While image-based filtering was found to be effective, it is crucial that it be limited to being applied in the direction orthogonal to the lines. Image-based filtering in the direction of the lines was detrimental to both accuracy and precision. In general, we found extracted line edge filtering (also referred to as post filtering) to be the more effective of the two methods, however, the best results are achieved by combining the two methods. Analysis of experimental data was also found to support the results based on modeling data.

The authors are greatly indebted to Paul Denham, Brian Hoef, Gideon Jones, Jerrin Chiu, and Ken Goldberg for expert support with the exposure tool, and to the entire CXRO staff for enabling this research. The authors are also grateful to Kim Dean of SEMATECH for program 
support. This research was supported by SEMATECH and carried out at Lawrence Berkeley National Laboratory under the auspices of the DOE Office of Basic Energy Science. 


\section{References}

1. International Technology Roadmap for Semiconductors, 2006 Update, (http://www.itrs.net/Links/2006Update/2006UpdateFinal.htm)

2. D. He, F. Cerrina, "Process dependence of roughness in a positive-tone chemically amplified resist,” J. Vac. Sci. \& Technol. B 16, 3748-3751 (1998).

3. V. Constantoudis, G. P. Patsis, A. Tserepi, E. Gogolides, "Quantification of line-edge roughness of photoresists. II. Scaling and fractal analysis and the best roughness descriptors,” J. Vac. Sci. \& Technol. B 21, 1019-1026 (2003).

4. B. Bunday, M. Bishop, D. McCormack, "Determination of optimal parameters for CD-SEM measurement of line-edge roughness," Proc. SPIE 5375, 515-533 (2004).

5. L. Liebovitch, J. Fischbargand and J. Koniarek "Ion channel kinetics: a model based on fractal scaling rather than multistate Markov processes," Math. Biosci. 84, 37-68 (1987).

6. V. Constantoudis, G. P. Patsis, L. Leunissen, E. Gogolides, "Line edge roughness and critical dimension variation: Fractal characterization and comparison using model functions,” J. Vac. Sci. \& Technol. B 22, 1974-1981 (2004).

7. G. Palasantzas, "Roughness spectrum and surface width of self-affine fractal surfaces via the kcorrelation model,” Phys. Rev. B 48, 14472-14478 (1993).

8. S. Prakash, S. Havlin, M. Schwartz, H. Stanley, "Structural and dynamical properties of longrange correlated percolation," Phys. Rev. A 46, R1724-R1727 (1992).

9. J. W. Goodman, Statistical Optics, John Wiley and Sons, New York, 1985, Chap. 5, 157-236.

10. P. Naulleau, K. Goldberg, E. Anderson, J. Cain, P. Denham, K. Jackson, A. Morlens, S. Rekawa, F. Salmassi, "EUV microexposures at the ALS using the 0.3-NA MET optic," J. Vac. Sci. \& Technol. B 22, 2962-2965 (2004). 


\section{List of Figures}

Fig. 1. Roughness exponent and correlation length extraction accuracy as a function of actual roughness exponent and correlation length using various data reduction methods. In (a) and (b) the actual correlation length is fixed at $25 \mathrm{~nm}$ while the roughness exponent is varied from 0.1 to 0.9 . In (c) and (d) the actual roughness exponent is fixed at 0.5 , while the correlation length is varied from 15 to 50 .

Fig. 2. Computed PSD with Gaussian noise added to the residual line edge data. The plots shows PSDs for two extremes of the added noise as well as the noise-free case. As is typically seen in experimental data, the plots with noise display an evident noise floor caused by the additive white Gaussian noise overriding the $1 / \mathrm{f}$ behavior of the self-affine edge data at high frequencies.

Fig. 3. Fractional error in the extracted roughness exponent (a) and absolute error in the extracted the correlation length (b) as a function of noise magnitude. The underlying data roughness exponent and correlation length are 0.75 and $25 \mathrm{~nm}$, respectively.

Fig. 4. Fractional error in the extracted roughness exponent (a) and absolute error in the extracted the correlation length (b) as a function of noise magnitude after filtering the residual lineedge data to remove all frequencies beyond the noise floor. The underlying data roughness exponent and correlation length are 0.75 and $25 \mathrm{~nm}$, respectively.

Fig. 5. Fractional precision in the extracted roughness exponent (a) and absolute precision in the extracted the correlation length (b) as a function of noise magnitude. Same filtering as used in Fig. 4 is used here. The underlying data roughness exponent and correlation length are 0.75 and $25 \mathrm{~nm}$, respectively. 
Fig. 6. Illustrative synthesized LER image comprised of a series of lines with self-affine edges with roughness exponent of 0.75 , correlation length of $25 \mathrm{~nm}$, and LER of $10 \mathrm{~nm}$. The line pitch is $270 \mathrm{~nm}$.

Fig. 7. LER PSD recovered upon analysis of 8 independent synthesized images. The images are generated to have a roughness exponent of 0.75 , correlation length of $25 \mathrm{~nm}$, and LER of $3 \mathrm{~nm}$.

Fig. 8. Representative synthesized image with LER and additive Gaussian noise where the standard deviation of the noise is $10 \%$ the full magnitude of the underlying synthesized image. The underlying data roughness exponent and correlation length are 0.75 and $25 \mathrm{~nm}$, respectively. Again the LER is set to $3 \mathrm{~nm}$.

Fig. 9. LER PSD corresponding to the analysis of 8 independent synthesized images as shown in Fig. 8. PSD displays noise floor behavior similar to that observed in the addition of edge noise (Fig. 2).

Fig. 10. Metric extraction accuracy as a function of added noise. The noise magnitude is defined as follows: a noise magnitude of 0.1 corresponds to the addition of Gaussian white noise with a standard deviation equal to $10 \%$ of the full magnitude of the noise-free lines. The underlying data roughness exponent and correlation length are 0.75 and $25 \mathrm{~nm}$, respectively. (a) and (b) show the results without any filtering and (c) and (d) show the results after filtering the residual line-edge data to remove all frequencies beyond the noise floor.

Fig. 11. Precision values as a function of noise for the image-noise case. (a) shows the fractional precision for the roughness exponent and (b) shows the absolute precision for the correlation length. As seen for noise added directly to the line-edge data, the precision is superior for the HHCF and SVL methods compared to the PSD method. 
Fig. 12. Roughness exponent extraction accuracy as a function of image-space filtering. The processed synthesized images are noise free. The plotted data is fractional error relative to the 0.75 underlying value for the roughness exponent.

Fig. 13. Correlation length extraction accuracy as a function of image-space filtering. The processed synthesized images are noise free. The plotted data is the extracted correlation length which should be compared to the $25 \mathrm{~nm}$ underlying value for the correlation length.

Fig. 14. Roughness exponent extraction accuracy as a function of image-space filtering. The processed synthesized images are noise free. The plotted data is fractional error relative to the 0.75 underlying value for the roughness exponent.

Fig. 15. Correlation length extraction accuracy as a function of image-space filtering. The processed synthesized images are noise free. The plotted data is the extracted correlation length which should be compared to the $25 \mathrm{~nm}$ underlying value for the correlation length.

Fig. 16. Roughness exponent extraction precision as a function of image-space filtering. The processed synthesized images include additive Gaussian white noise with a standard deviation equal to $5 \%$ of the full magnitude of the noise-free lines. The plotted data is fractional precision relative to the average extracted value for the roughness exponent.

Fig. 17. Correlation length extraction precision as a function of image-space filtering. The processed synthesized images include additive Gaussian white noise with a standard deviation equal to $5 \%$ of the full magnitude of the noise-free lines. The plotted data is absolute precision correlation length.

Fig. 18. Comparison of the effectiveness of extracted line-edge data and image-space filtering. (a) and (b) show the roughness exponent and correlation length extraction accuracy as a function of 
noise with image-space filtering alone whereas (c) and (d) show the results when combined with lineedge data filtering. The image-space filter is $12 \times 1$ pixels (orthogonal to lines) with a $\sigma$ of 4 .

Fig. 19. Precision values as a function of noise for the image-noise case when both iamgespace and line-edge data filtering are used. The image-space filter is $12 \times 1$ pixels (orthogonal to lines) with a $\sigma$ of 4. (a) shows the fractional precision for the roughness exponent and (b) shows the absolute precision for the correlation length.

Fig. 20. Representative SEM image from the experimental dataset. Images are of $60 \mathrm{~nm}$ lines and space in resist printed using a 0.3-NA EUV exposure tool. The images are taken at a magnification of 150,000 .

Fig. 21. LER PSD averaged over the entire experimental dataset (1030 edges). A significant noise floor is evident arising from metrology noise, which can be observed as typical SEM "snow" in Fig. 20. 


\section{List of Tables}

Table 1. Metrics extracted from the 100 image (1030 edge) experimental dataset. Prefilt corresponds to image-space filtering and Postfilt to extracted line-edge data filtering. Filter setting values of 1 indicate the filter was used and values of 0 indicate that it was not. When used, the imagespace filter is $12 \times 1$ pixels (orthogonal to lines) with a $\sigma$ of 4 .

Table 2. Single image metric extraction precision for the experimental dataset. The precision values for the roughness exponent are fractional relative to the 100 -image averaged value and the correlation length precision values are absolute. 
(a)

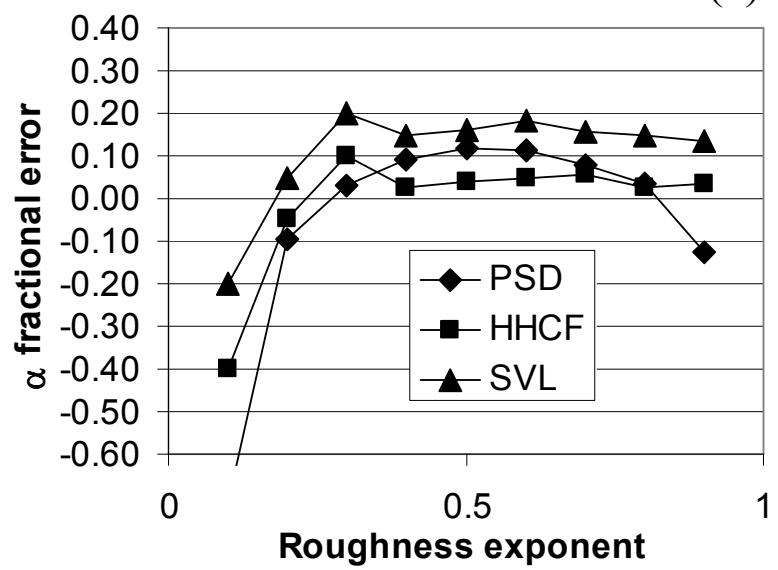

(c)

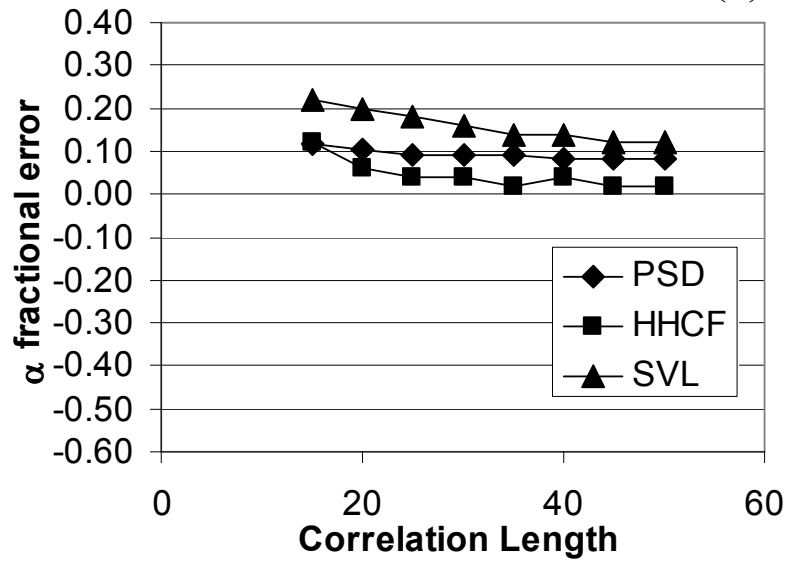

(b)

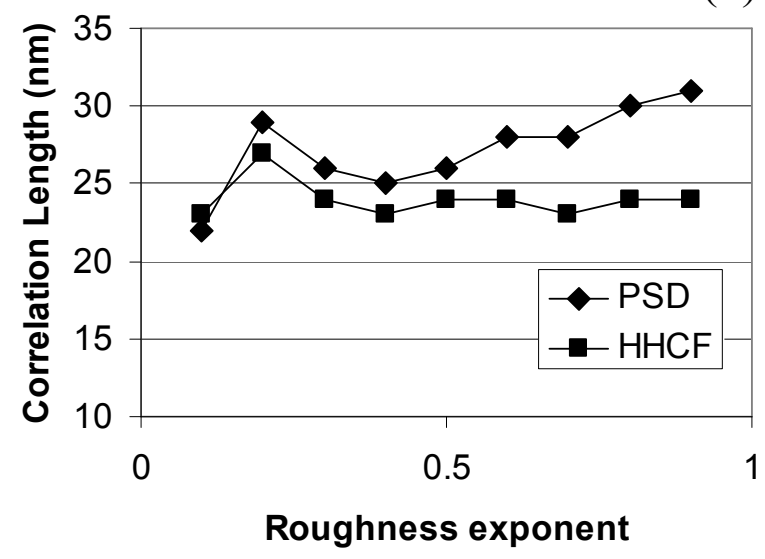

(d)

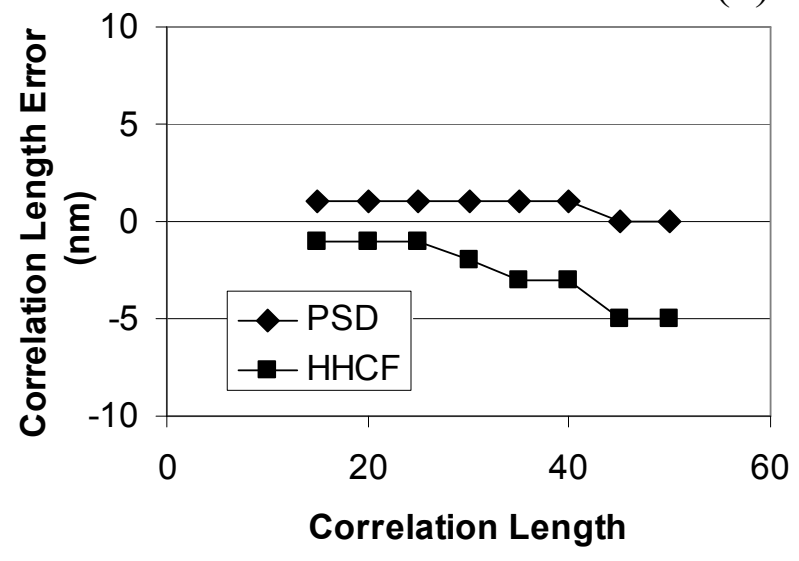


Naulleau, et al., Fig. 2

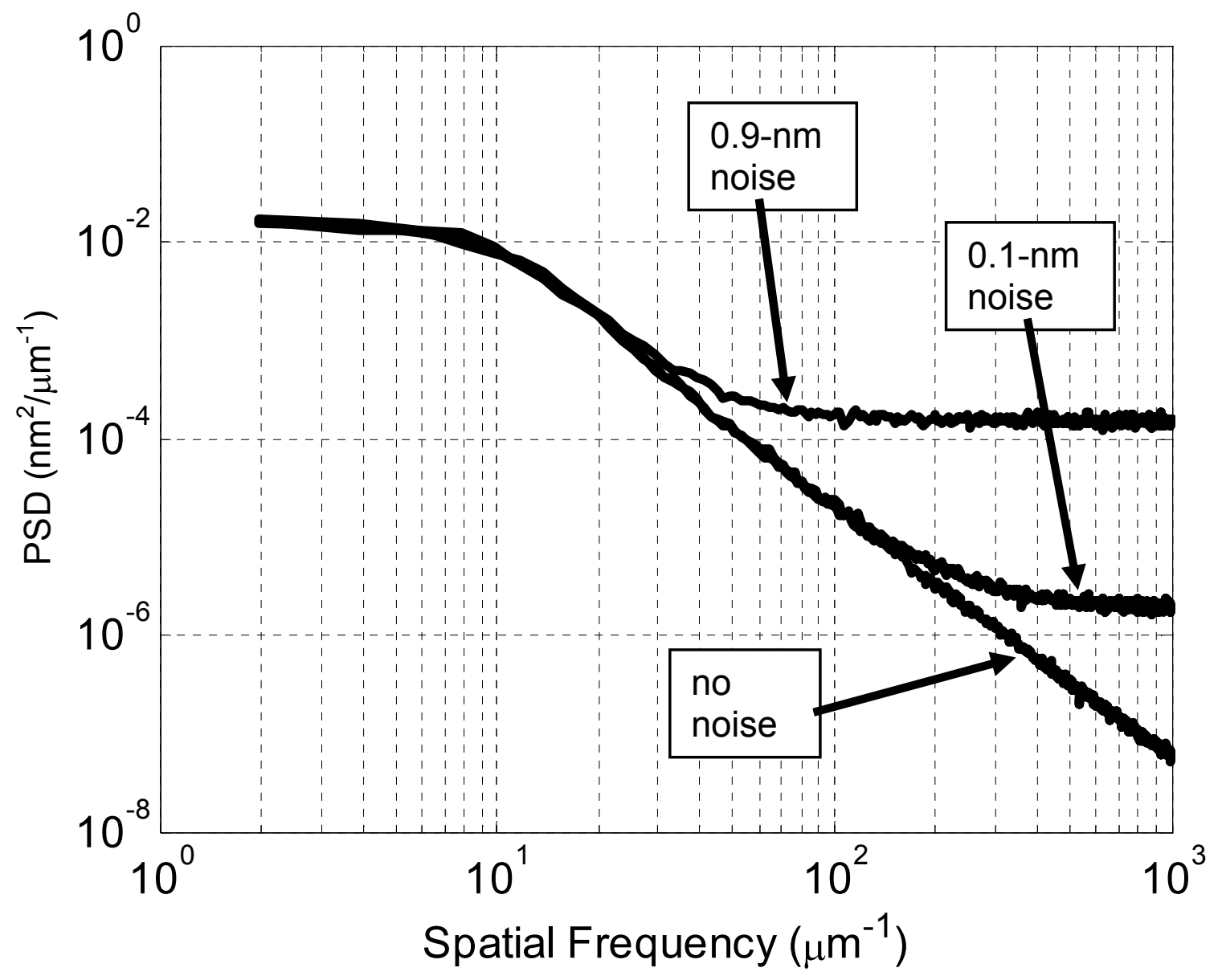


Naulleau, et al., Fig. 3

(a)

(b)
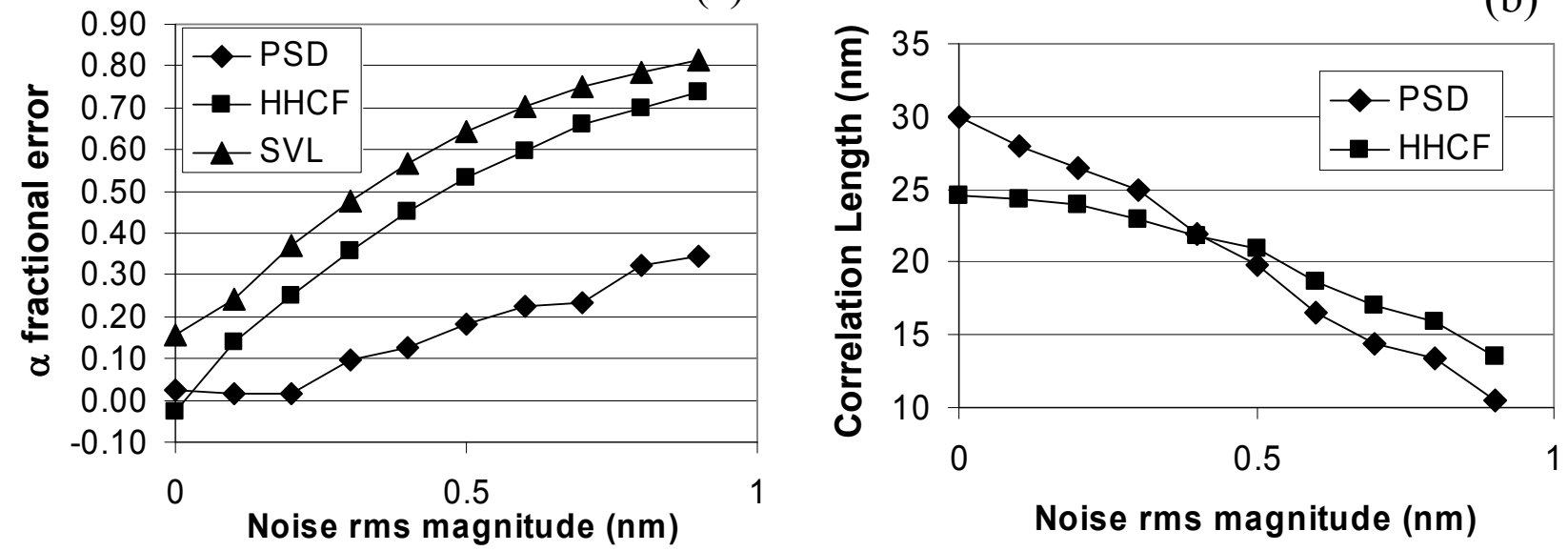
Naulleau, et al., Fig. 4

(a)

(b)
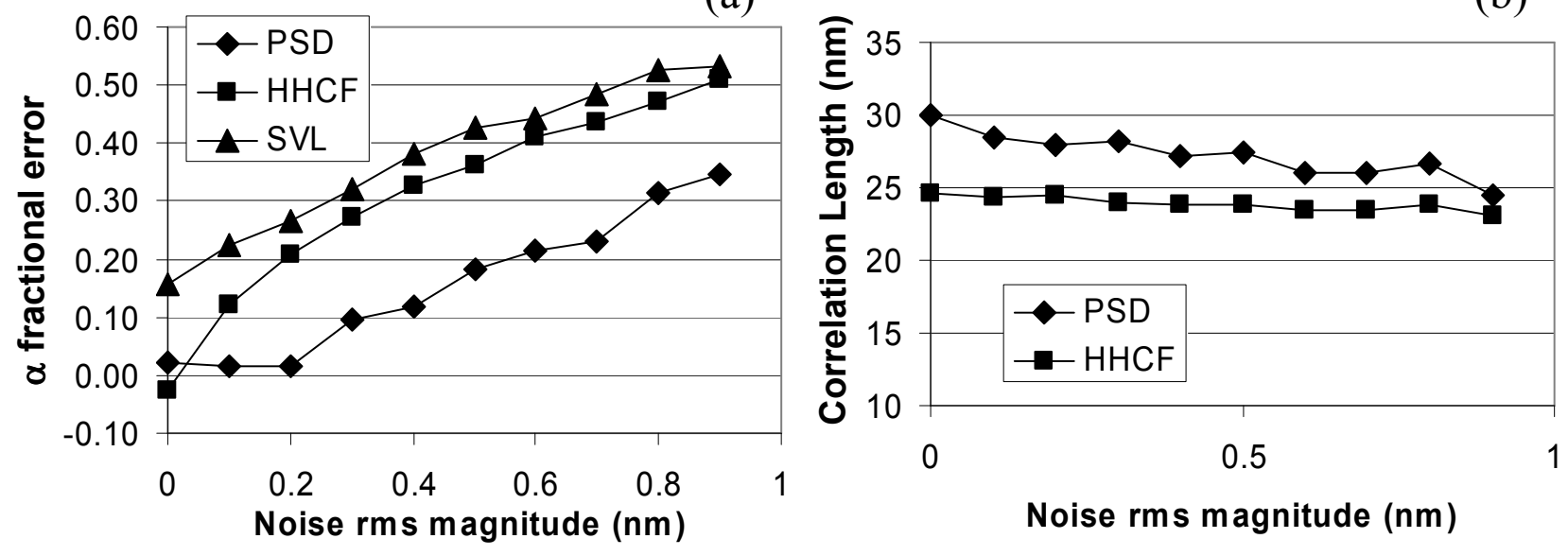
Naulleau, et al., Fig. 5

(a)

(b)
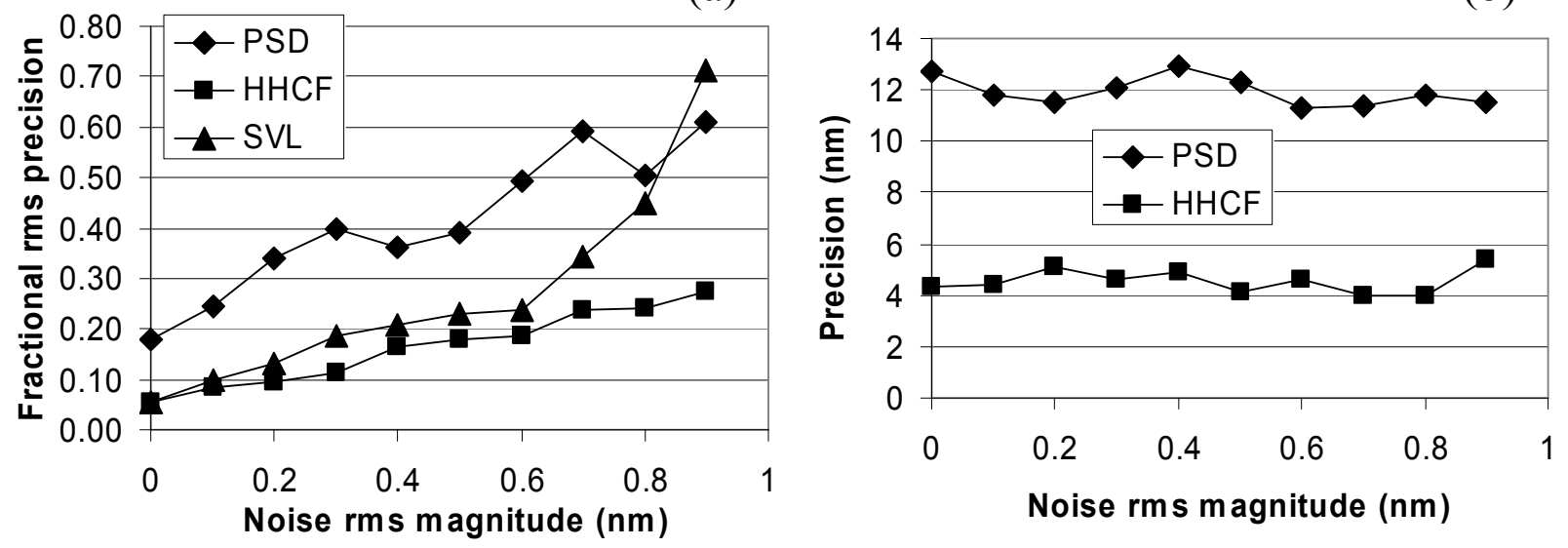


$$
\text { Naulleau, et al., Fig. } 6
$$

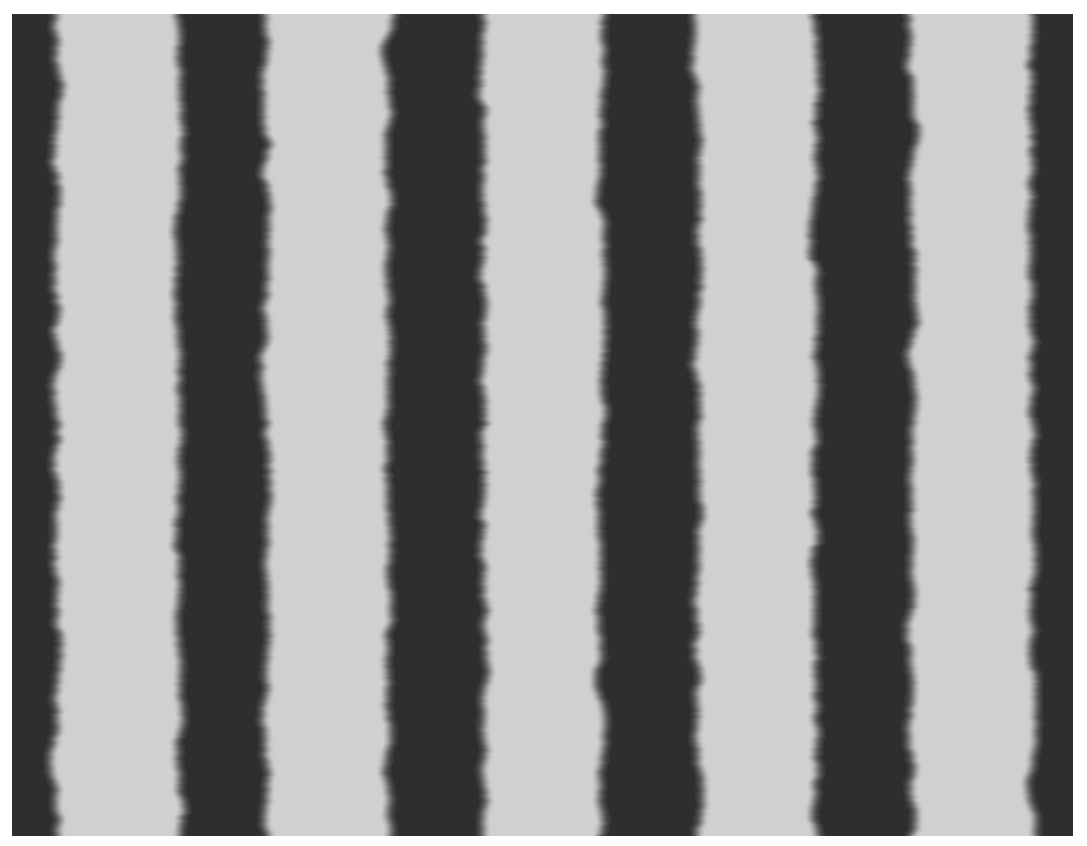


Naulleau, et al., Fig. 7

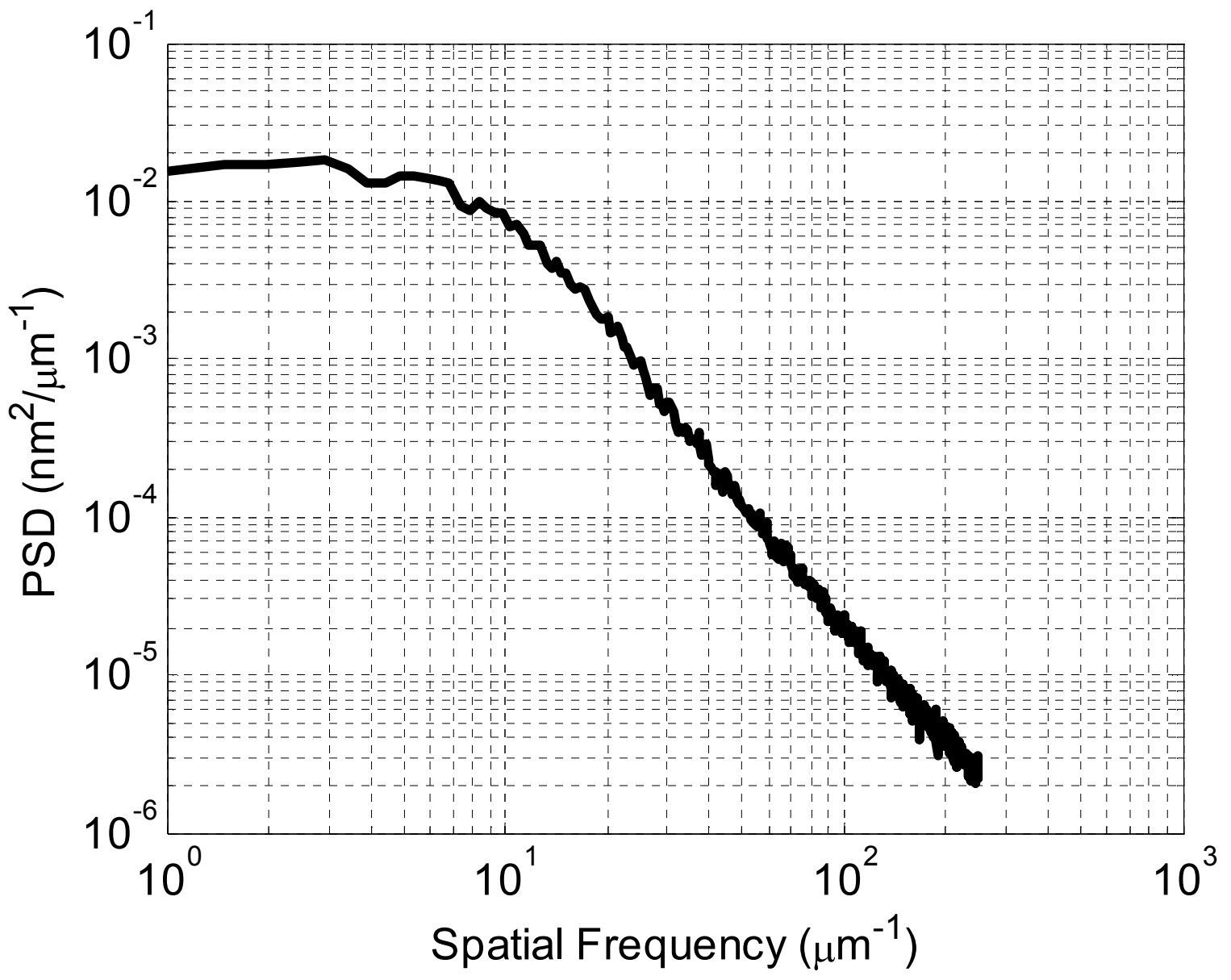


Naulleau, et al., Fig. 8

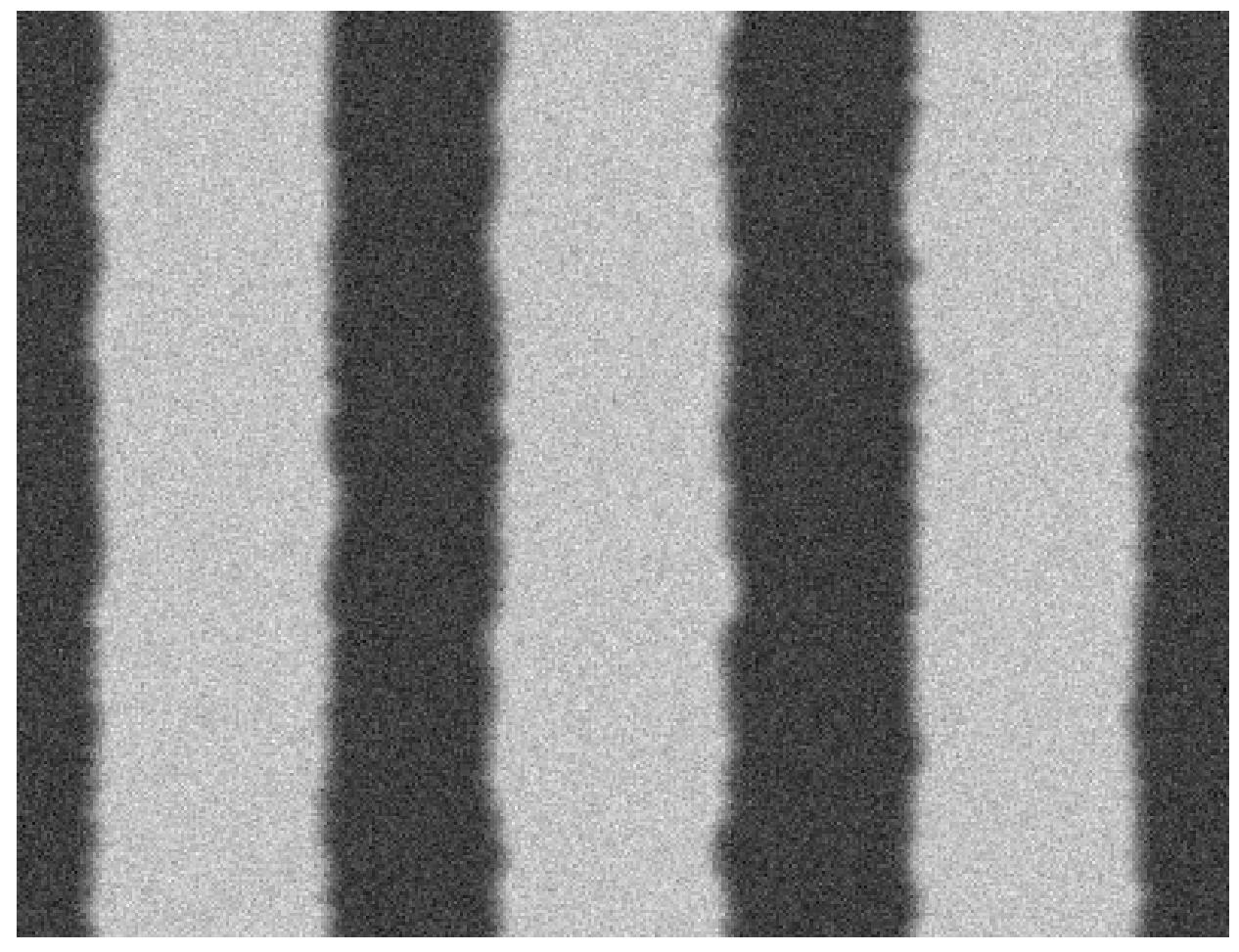


Naulleau, et al., Fig. 9

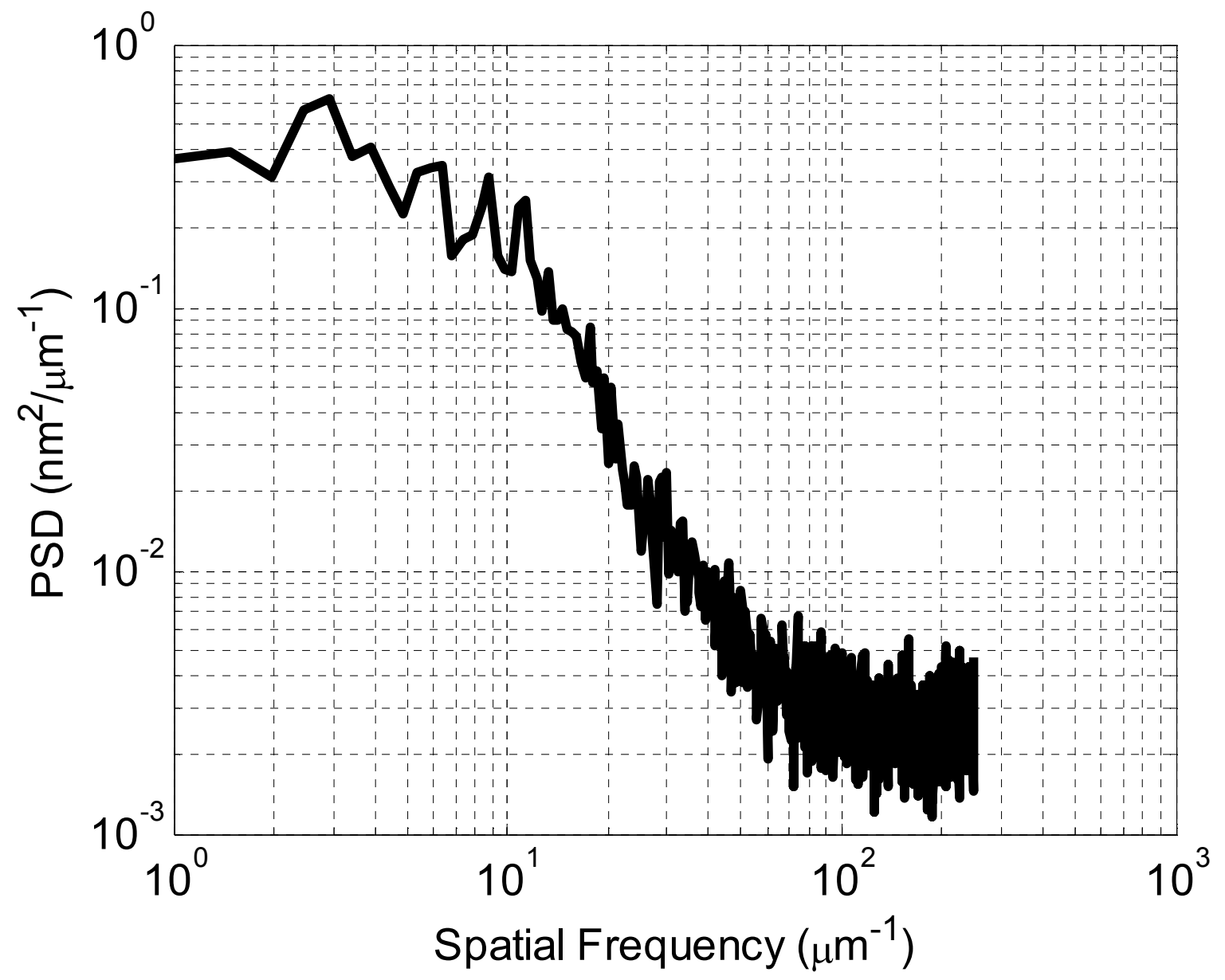


Naulleau, et al., Fig. 10

(a)

(b)
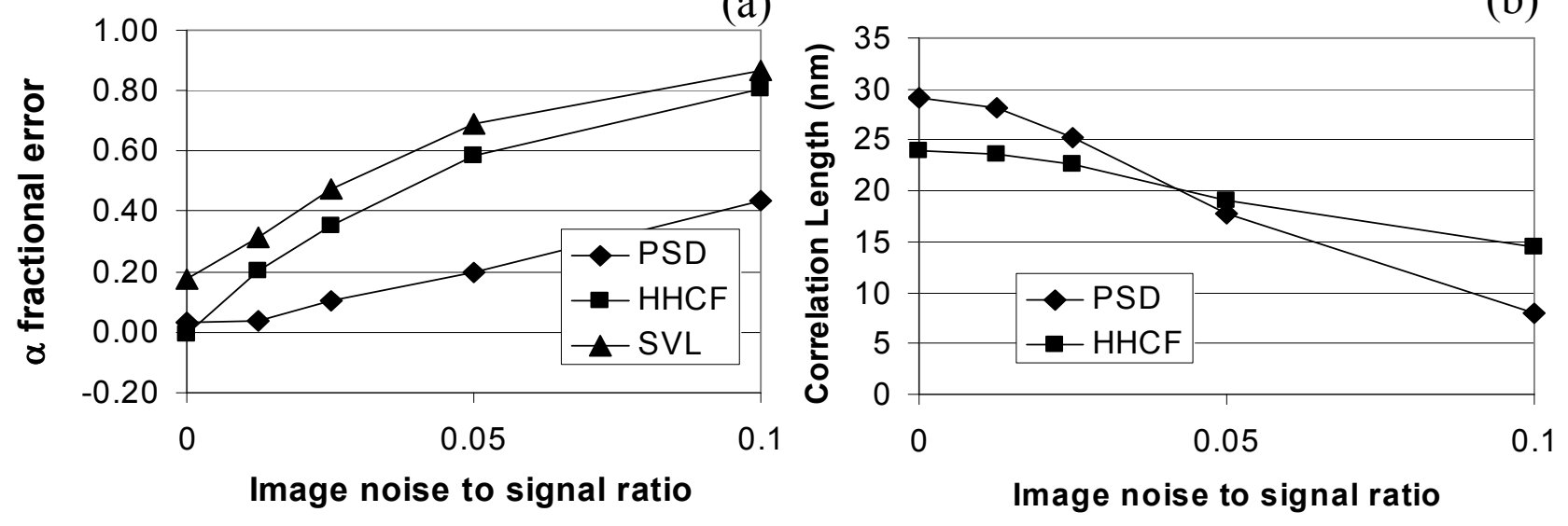

(c)

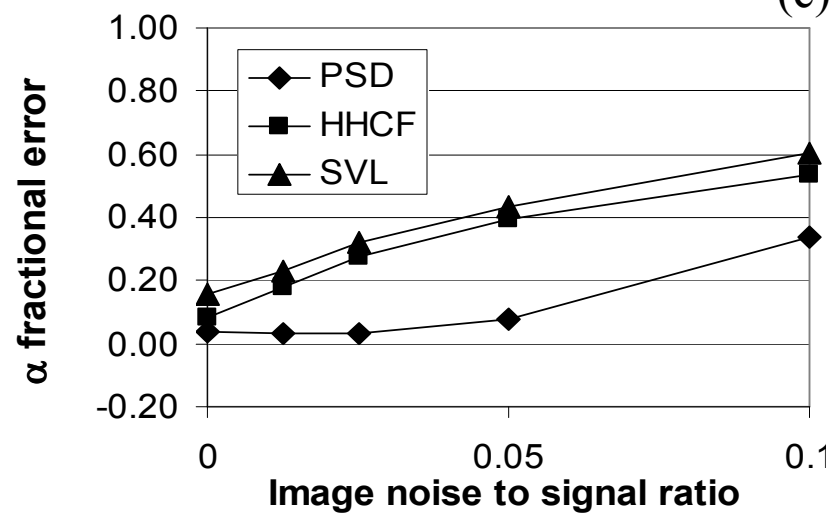

(d)

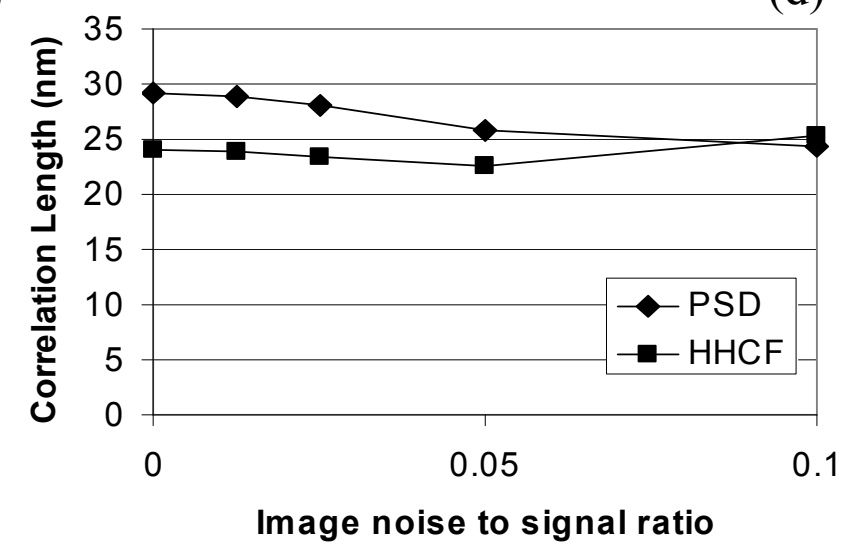


Naulleau, et al., Fig. 11

(a)

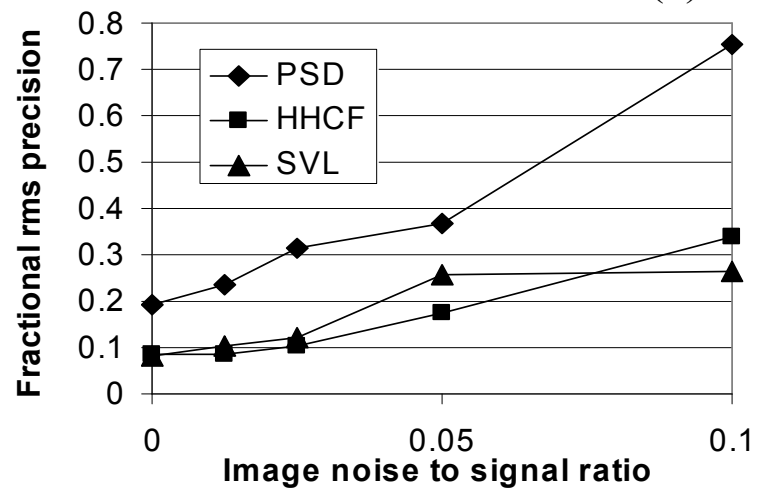

(b)

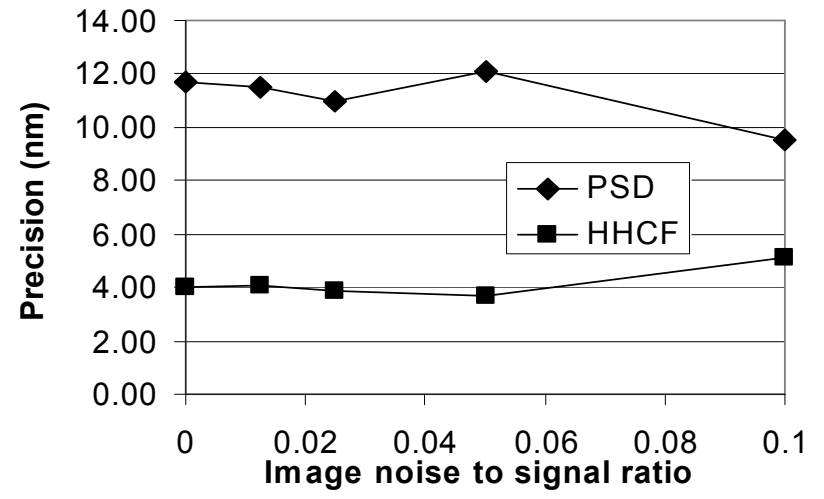



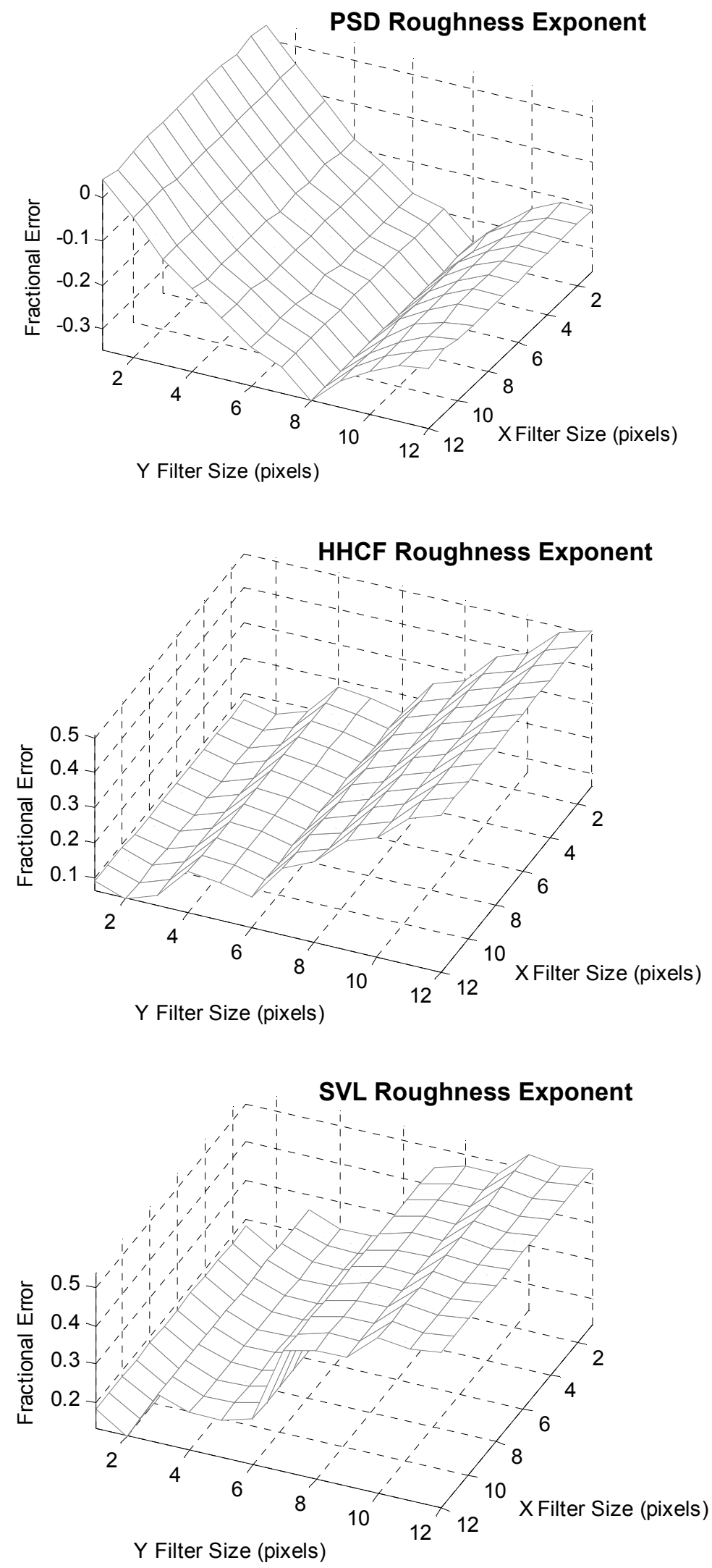
Naulleau, et al., Fig. 13
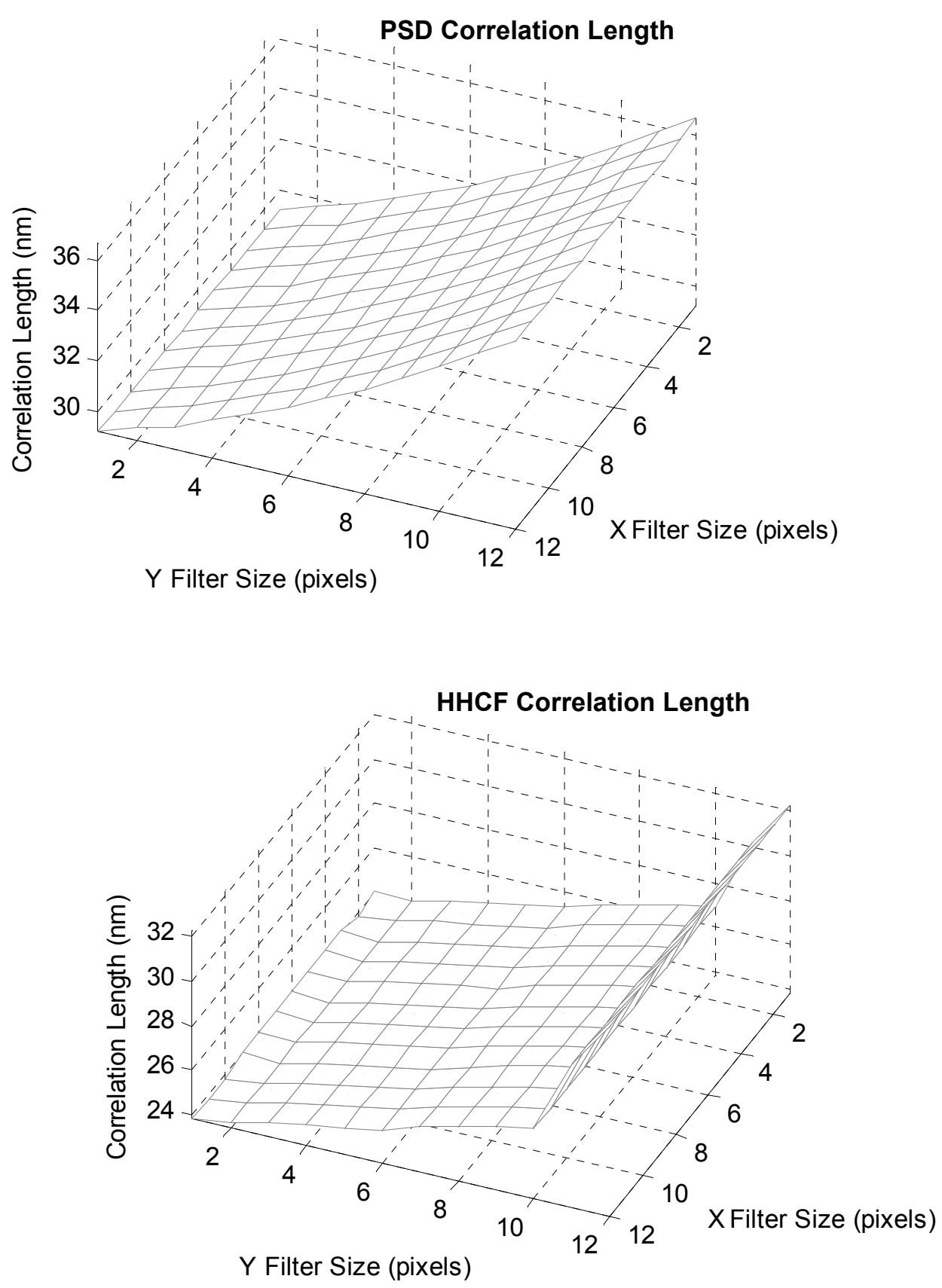

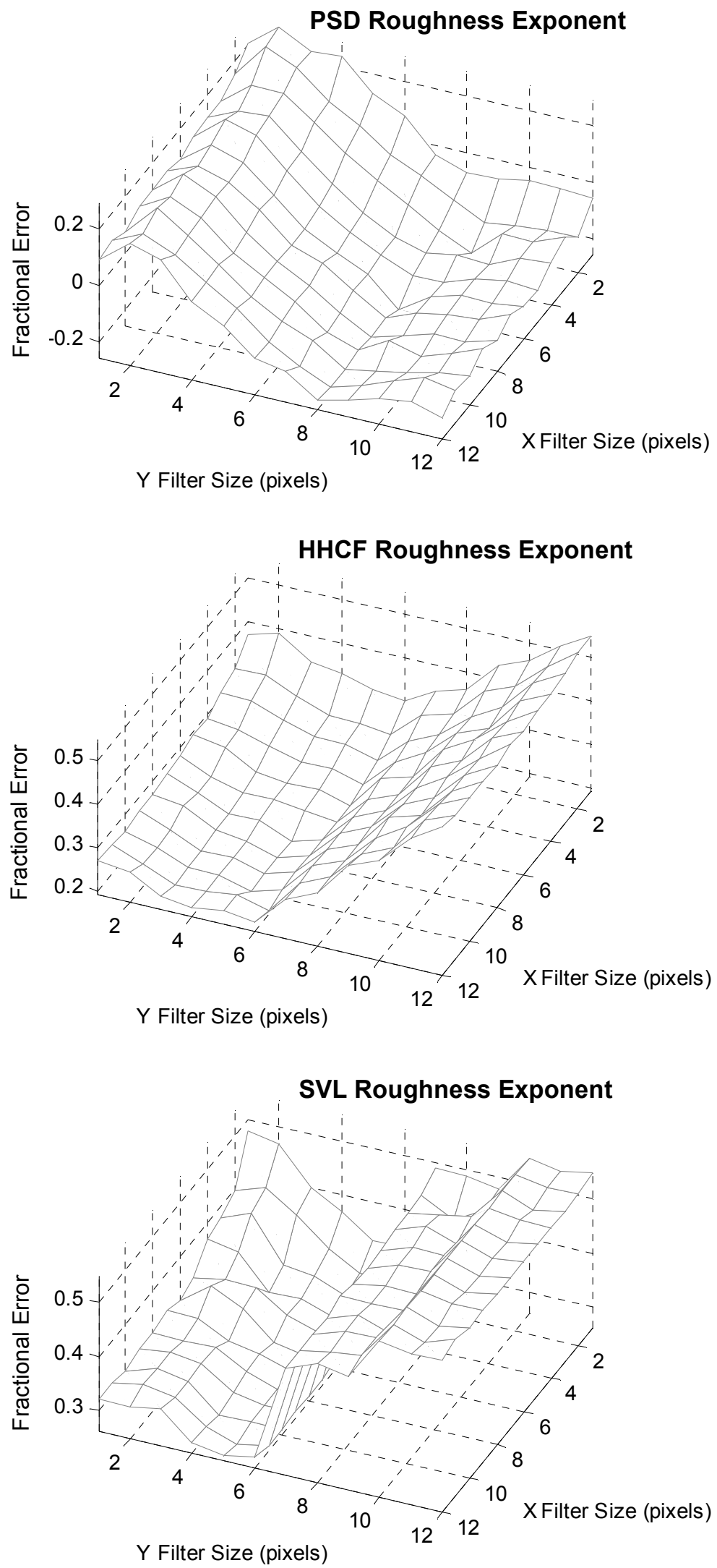
Naulleau, et al., Fig. 15
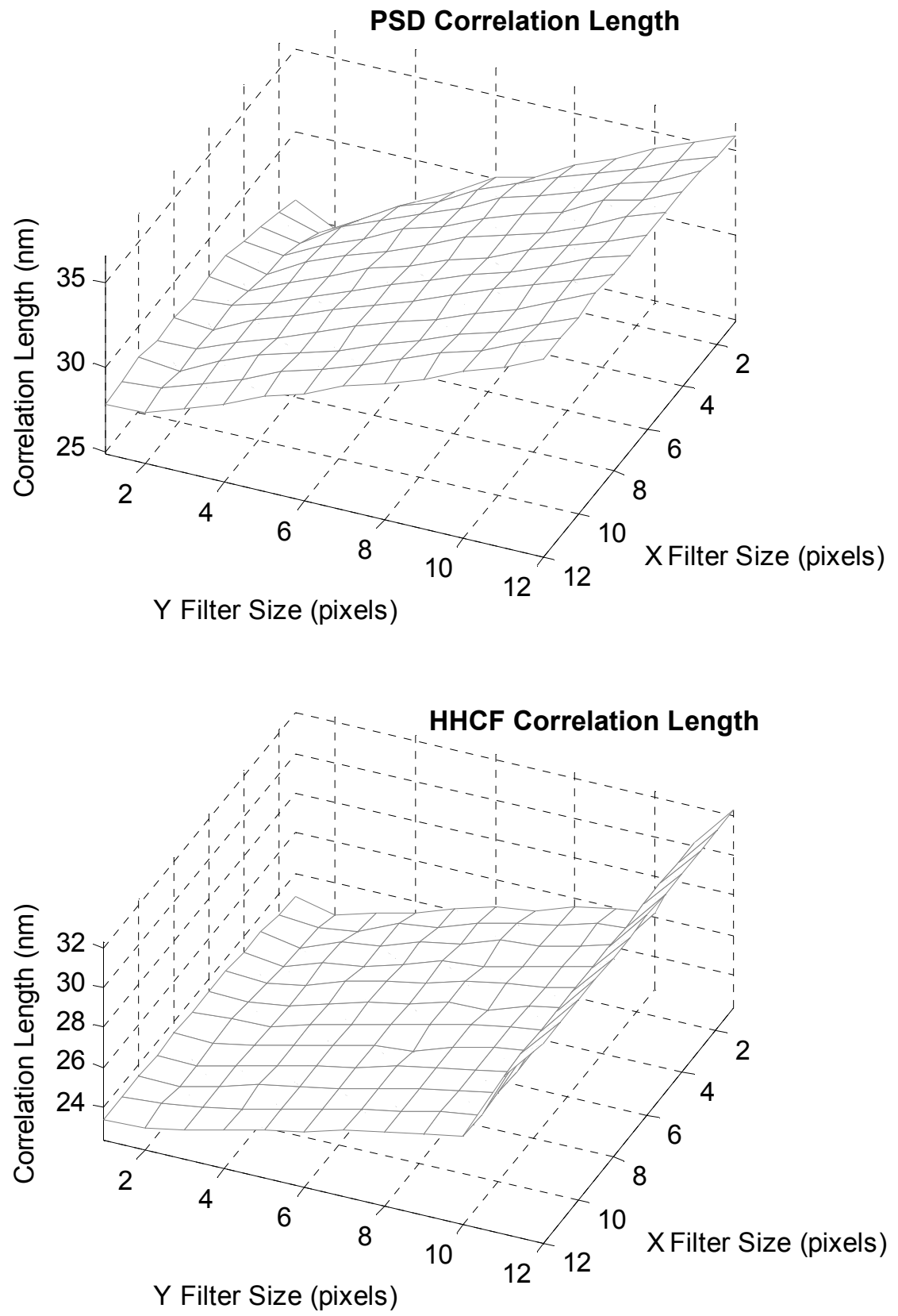

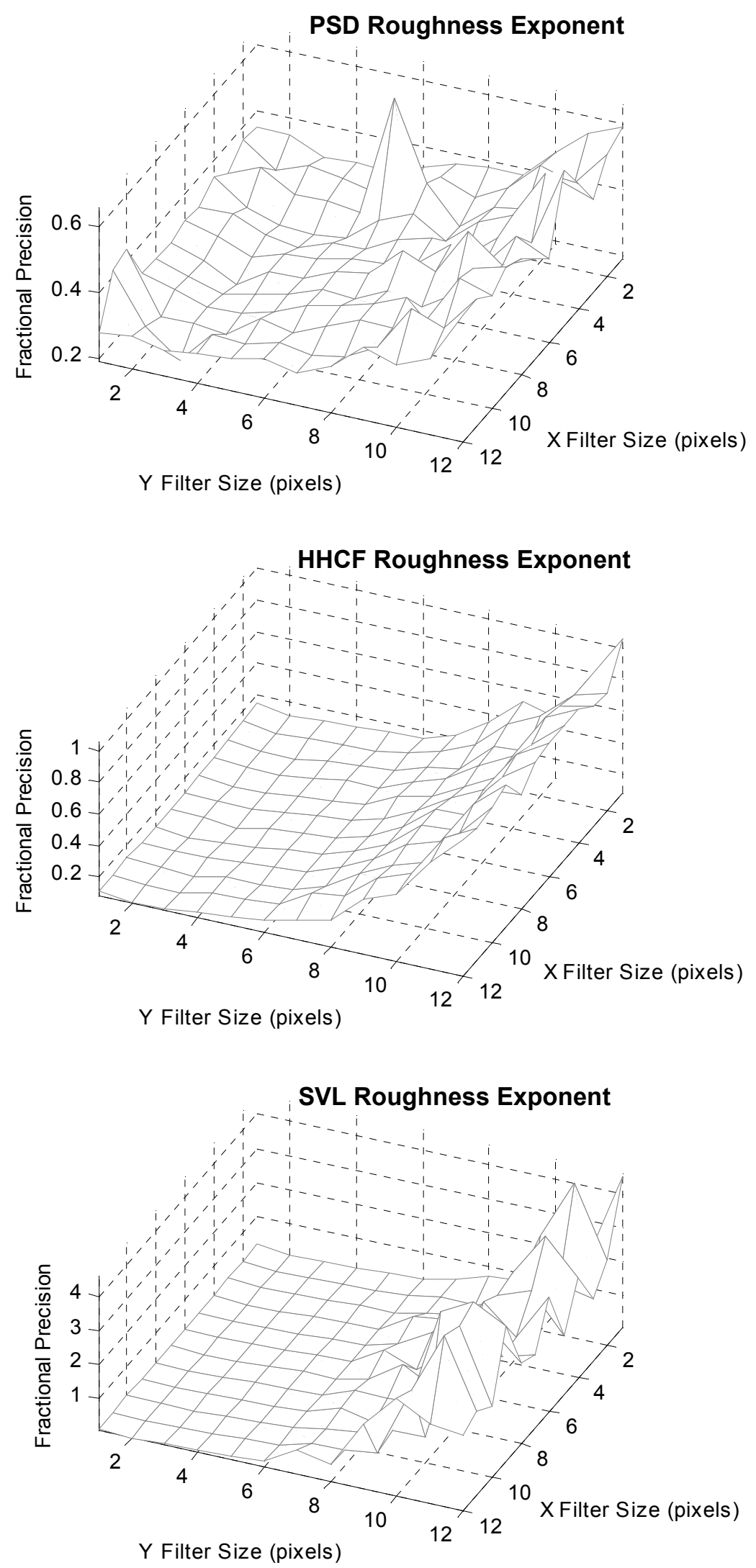
Naulleau, et al., Fig. 17
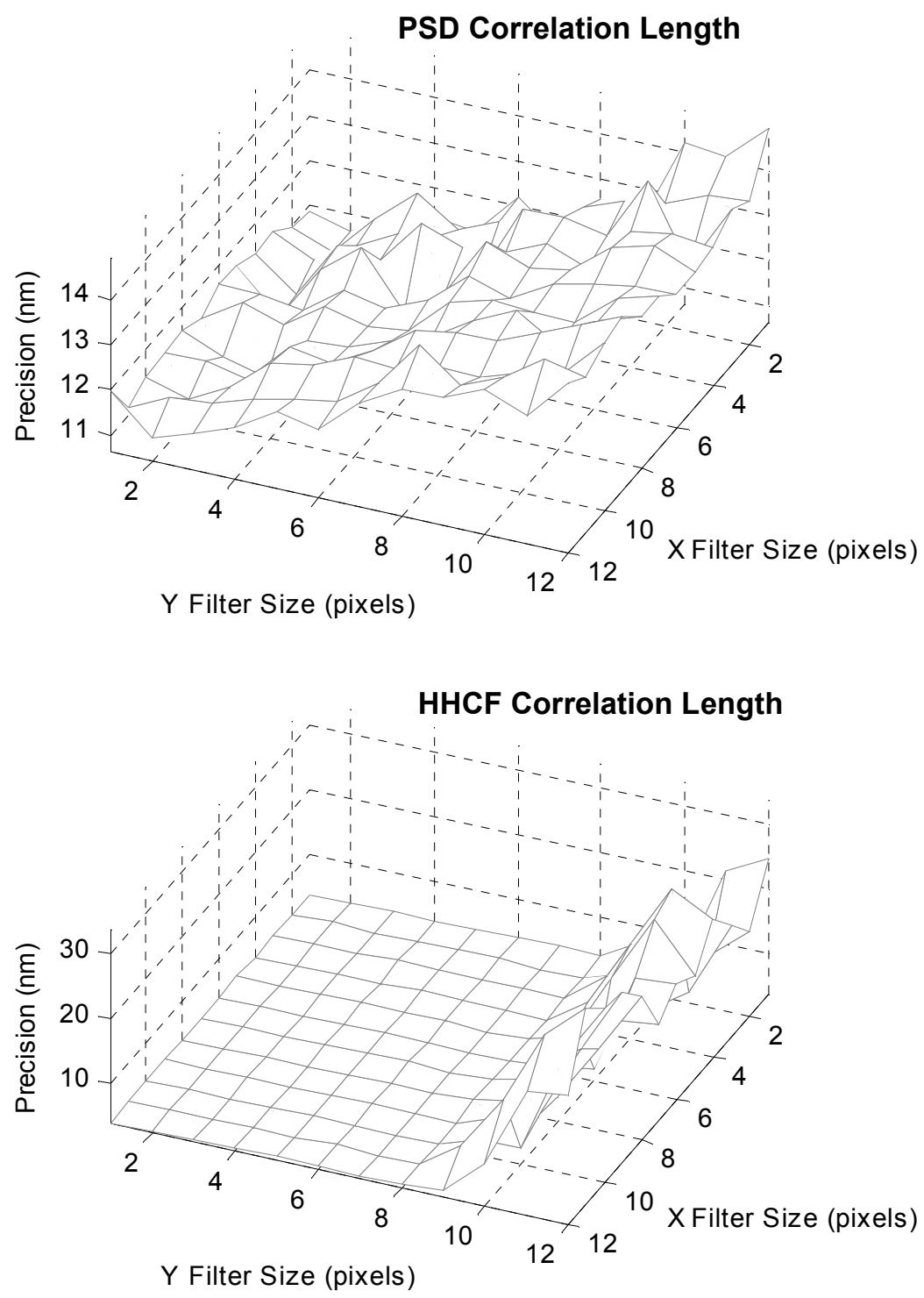
Naulleau, et al., Fig. 18

(a)

(b)
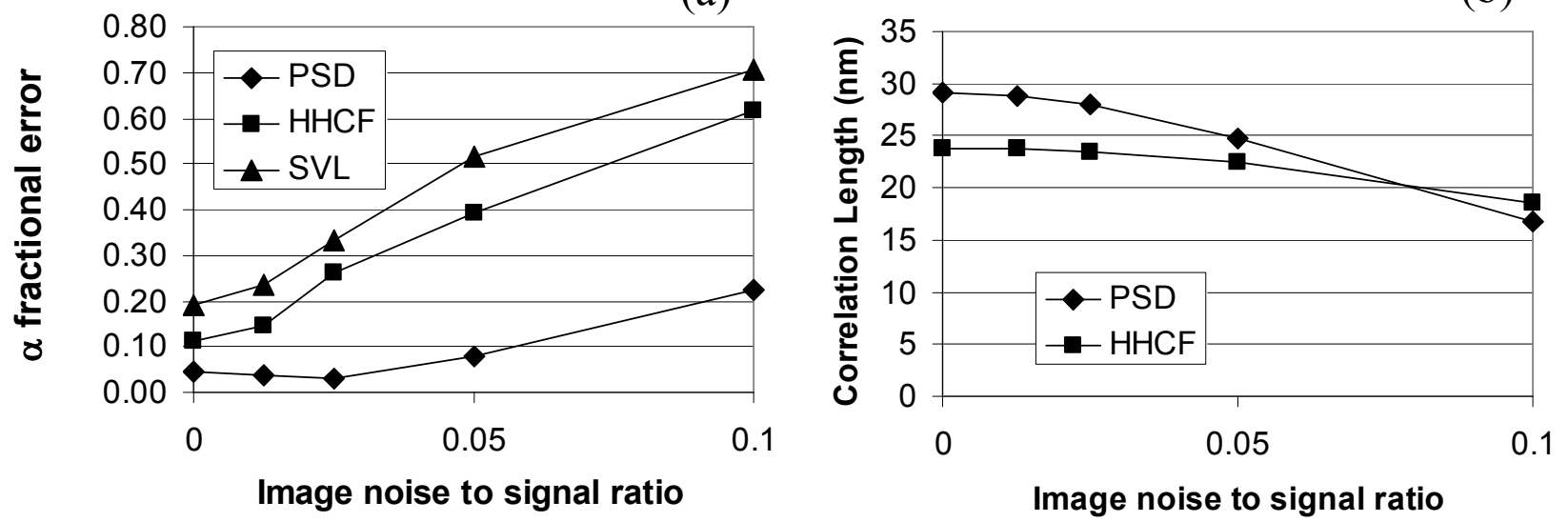

(c)

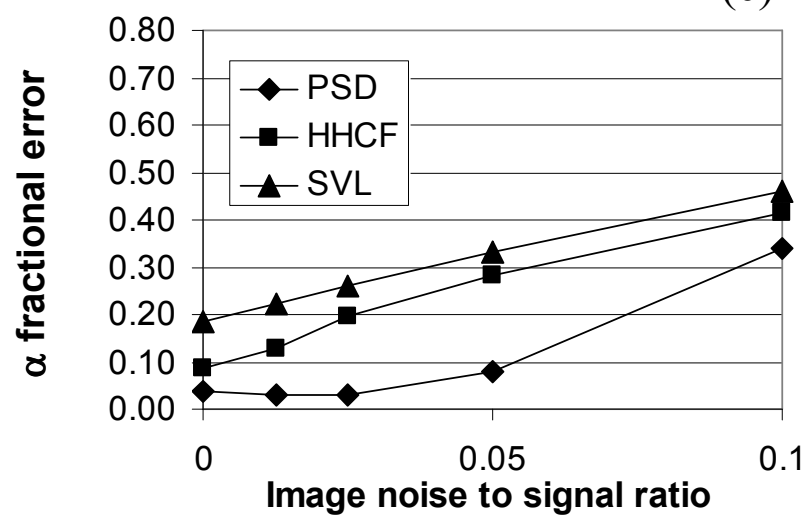

(d)

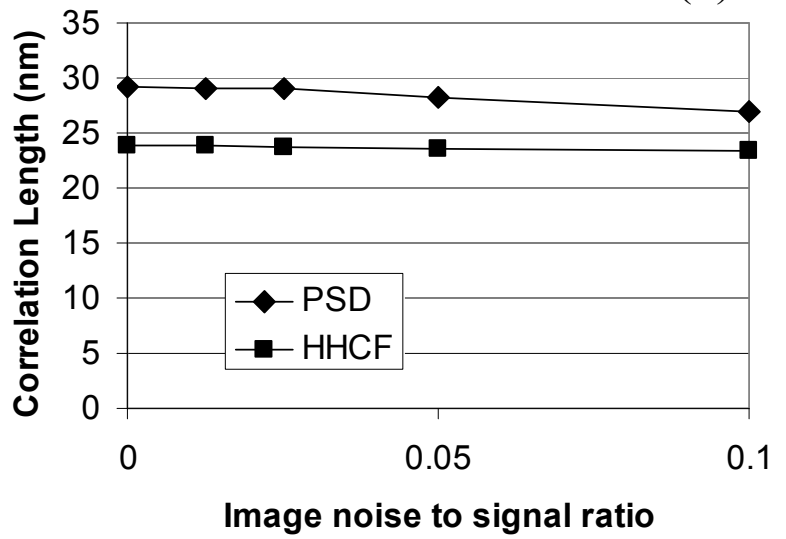


Naulleau, et al., Fig. 19

(a)

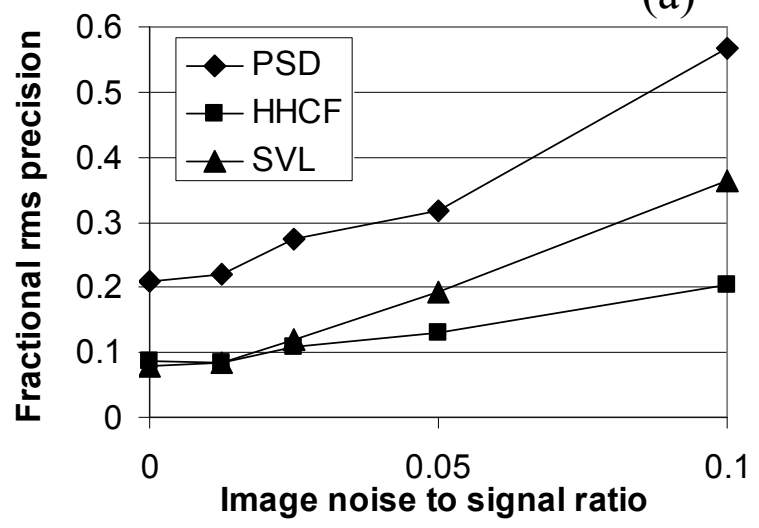

(b)

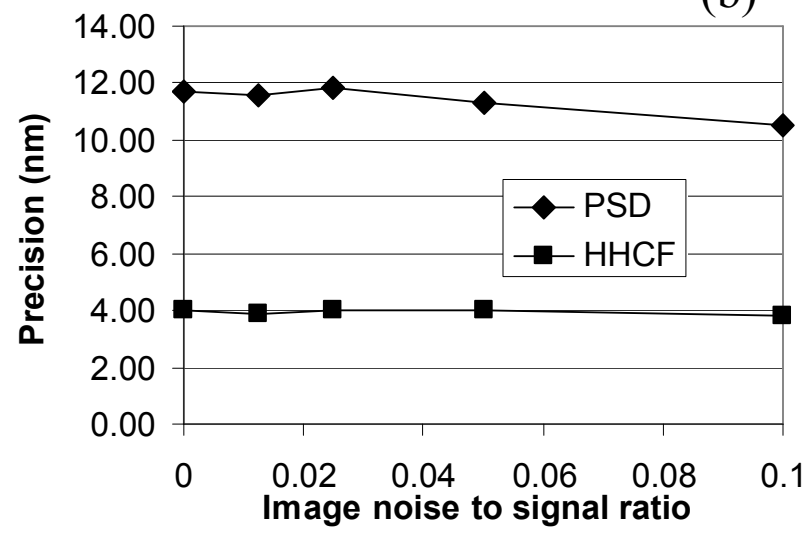


Naulleau, et al., Fig. 20

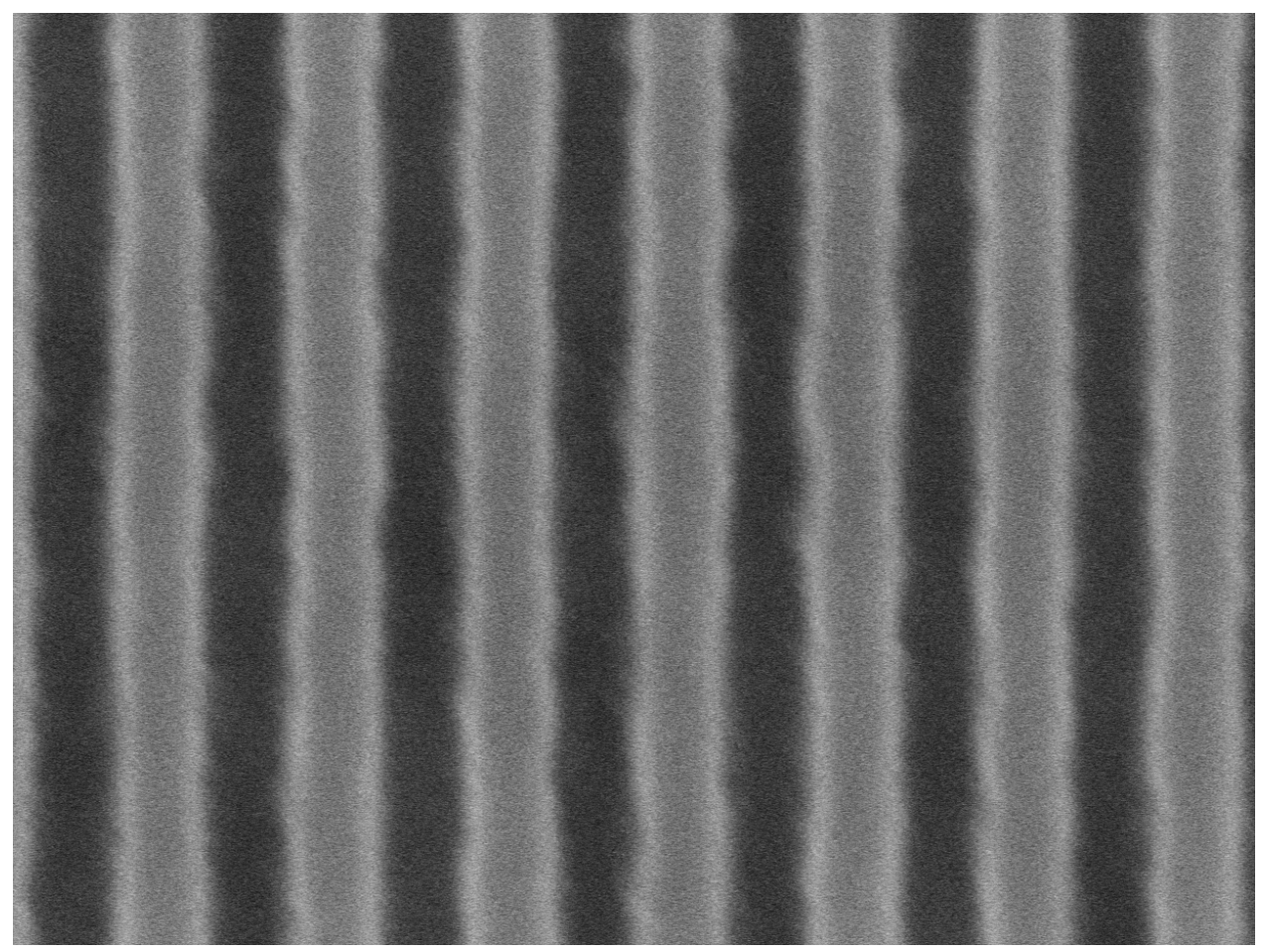


Naulleau, et al., Fig. 21

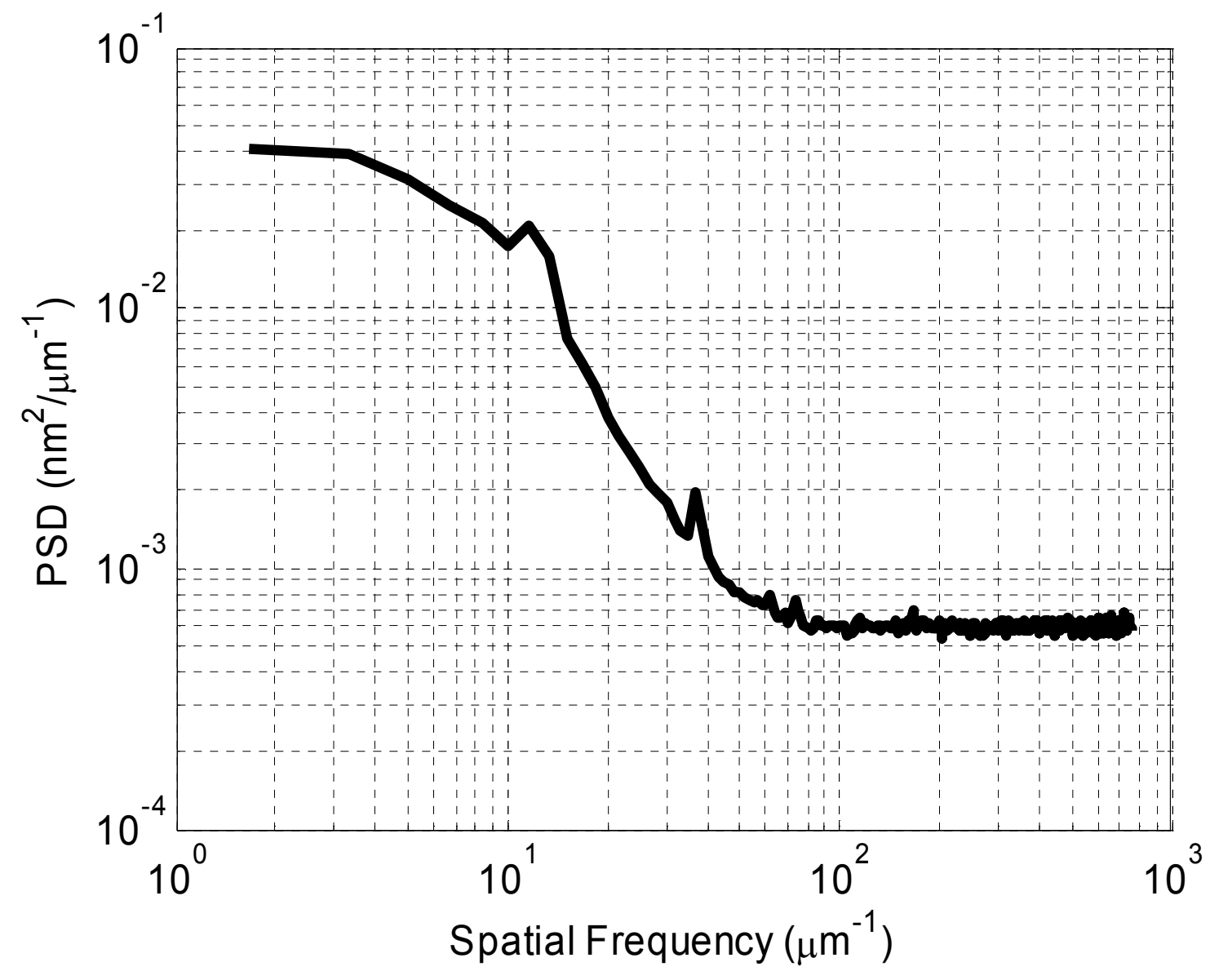


Naulleau, et al., Table 1

\begin{tabular}{|c|c|c|c|c|c|c|}
\hline \multicolumn{2}{|c|}{ Filter Settings } & \multicolumn{2}{c|}{ PSD } & \multicolumn{2}{c|}{ HHCF } & SVL \\
\hline Prefilt & Postfilt & $\boldsymbol{\alpha}$ & $\mathbf{L}_{\mathbf{c}}(\mathbf{n m})$ & $\boldsymbol{\alpha}$ & $\mathbf{L}_{\mathbf{c}}(\mathbf{n m})$ & $\boldsymbol{\alpha}$ \\
\hline 0 & 0 & 0.53 & 7.41 & 0.17 & 12.58 & 0.13 \\
\hline 0 & 1 & 0.53 & 25.05 & 0.46 & 20.96 & 0.45 \\
\hline 1 & 0 & 0.65 & 16.33 & 0.33 & 18.26 & 0.22 \\
\hline 1 & 1 & 0.65 & 28.39 & 0.52 & 22.14 & 0.51 \\
\hline
\end{tabular}


Naulleau, et al., Table 2

\begin{tabular}{|c|c|c|c|c|c|c|}
\hline \multicolumn{2}{|c|}{ Filter Settings } & \multicolumn{2}{c|}{ PSD } & \multicolumn{2}{c|}{ HHCF } & SVL \\
\hline Prefilt & Postfilt & $\boldsymbol{\alpha}$ & $\left.\mathrm{L}_{\mathbf{c}} \mathbf{( n m}\right)$ & $\boldsymbol{\alpha}$ & $\left.\mathbf{L}_{\mathbf{c}} \mathbf{( n m}\right)$ & $\boldsymbol{\alpha}$ \\
\hline 0 & 0 & 0.56 & 3.02 & 0.23 & 1.44 & 0.22 \\
\hline 0 & 1 & 0.63 & 4.52 & 0.12 & 2.38 & 0.17 \\
\hline 1 & 0 & 0.29 & 4.20 & 0.13 & 2.11 & 0.20 \\
\hline 1 & 1 & 0.28 & 4.64 & 0.09 & 2.47 & 0.13 \\
\hline
\end{tabular}

